\title{
A Case of Recurrent Gastrointestinal Stromal Tumour (Gist): Case Report and Review of Literature
}

\author{
Authors \\ Vikrant $\mathbf{S} \mathbf{N}^{1}$, Mohan Kumar $K^{2}$, Suma $S^{3}$ \\ Department of General Surgery \\ Sri Devaraj Urs Medical College, Kolar, India
}

\section{Introduction}

Gastrointestinal stromal tumors (GIST) are the most frequently encountered mesenchymal neoplasms of the gastrointestinal tract. They can also originate in the mesentery and omentum ${ }^{1}$. Incidence wise, they are rare and rank a distant third after adenocarcinoma and lymphomas among the histologic types of gastrointestinal tract tumors.

Historically, these lesions were classified as leiomyomas or leiomyosarcomas because they possessed smooth muscle features when examined under light microscopy. GISTs are usually found in adults between ages 40 and 70 and can be cancerous or noncancerous ${ }^{2}$.

Here we present a case report of a $65 \mathrm{yr}$ old male patient who presented to our outpatient with complaints of a recurrent mass in upper abdomen of 2 months duration. On examination a solitary, tender mass of size $20 \times 15 \mathrm{~cm}$ occupying left hypochondrium, lumbar and umbilical regions, having firm to hard consistency, with nodular surface was noted.

GIST traditionally has been viewed as a rare, enigmatic, and unpredictable tumor capable of aggressive behavior, and surgery has been the mainstay of treatment ${ }^{3}$.
Recurrence of GIST is fairly uncommon and Imatininib mesylate remains the drug of choice ${ }^{3}$.

\section{Case Report}

A $65 \mathrm{yr}$ old male patient presented to our outpatient department with complaints of mass in upper abdomen of 2 months duration, loss of appetite and weight loss of 1 month duration with associated abdominal pain of 15 days. Patient gives history of undergoing laparotomy in 2009 for a similar mass in the abdomen. No notable co morbidities were present.

On examination a solitary, tender mass of size $20 \times 15 \mathrm{~cm}$, occupying left hypochondrium, lumbar and umbilical regions having firm to hard in consistency, with nodular surface was noted. The mass showed mobility with respiration. A lower midline laparotomy scar measuring $10 \mathrm{~cm}$ was seen on the abdominal wall, healed with primary intention and healthy.Rest of the abdomen and per rectal examination were normal. 


\section{JMSCR Vol||05||Issue||07||Page 24767-24772||July}

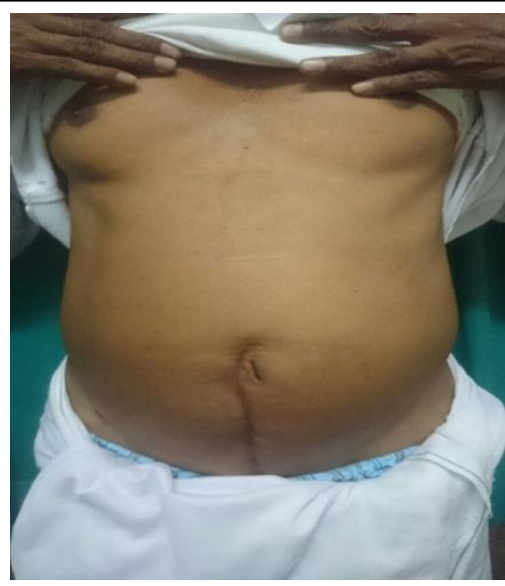

Preoperative picture

\section{Investigations}

Ultrasound of the abdomen and pelvis revealed a large multiloculated and complex predominantly cystic mass with vascular soft tissue components in the left lower abdomen/ flank region.

Contrast enhanced computerised tomography (CECT) of the abdomen and pelvis revealed a large well defined lobulated and thick peripherally enhancing fluid attenuation mesenteric mass lesion, likely cystic with a necrotic core, with multiple well defined heterogeneously enhancing nodular mesenteric/ Omental masses, comprising of cystic and enhancing solid components; along with focal enhancement and wall thickening, involving left anterolateral wall of rectum with few clusters of nodular densities in adjacent perirectal fat planes.
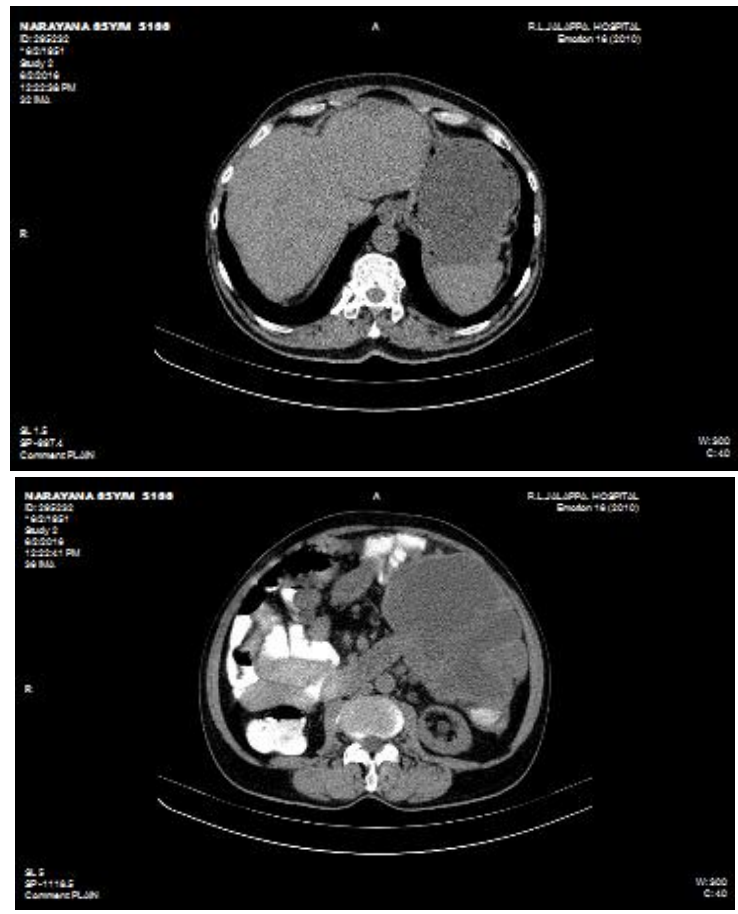
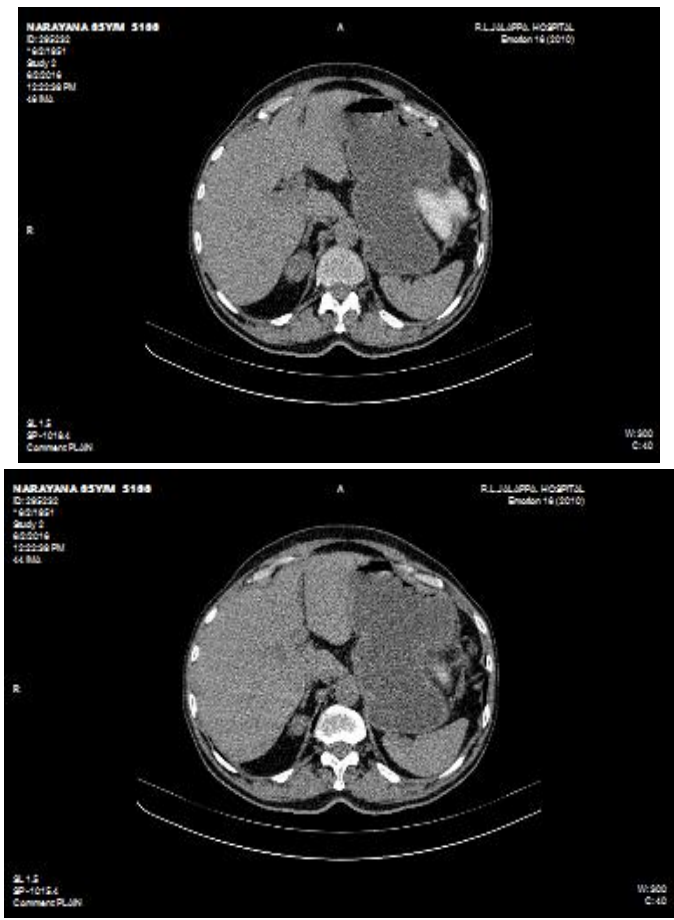

Colonoscopy was performed in view of the CECT finding which showed a normal study.

Chest X-ray revealed no significant abnormalities. Blood investigations including hemogram, electrolytes and liver function tests were within normal limits.

Exploratory laparotomy was performed; intraoperatively a $22 \mathrm{~cm} \times 15 \mathrm{~cm}$ hard mass with nodular surface arising from the mesentery of jejunum, infiltrating the descending colon, splenic flexure of colon, hilum of spleen and left kidney with multiple peritoneal metastases were seen. The tumour was unresectable, hence the procedure was abandoned. Biopsy was taken from peritoneal deposits and the tumour Medical oncologist opinion was sought suggesting Imatinib Mesylate therapy.

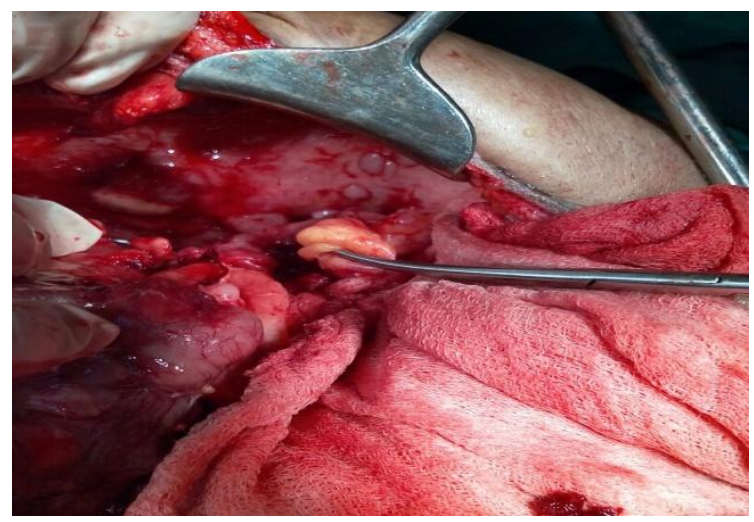




\section{JMSCR Vol||05||Issue||07||Page 24767-24772||July}

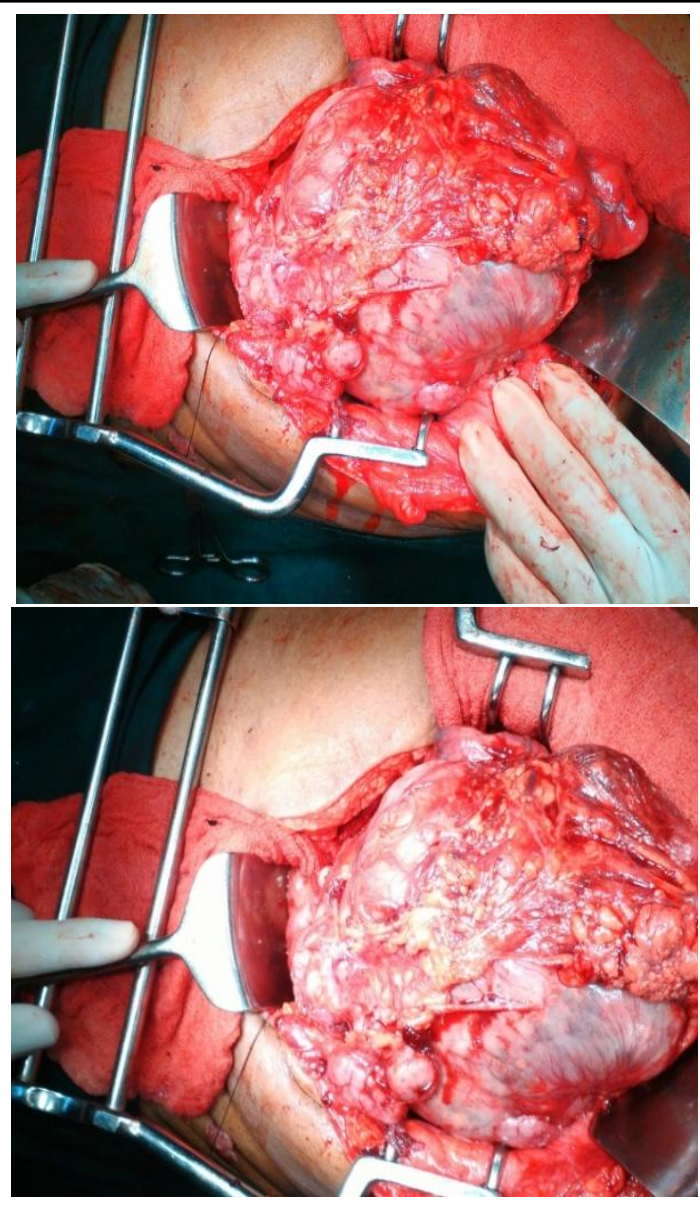

Intraoperative Images

Histopathology showed neoplasm composed of spindle cells arranged in sheets, with a whorled pattern. Tumour cells show pleomorphic nuclei with mitotic activities (> 10/10 HPR). At few places tumor cells show moderate amount of eosinophilic cytoplasm and vacuolated cytoplasm. Tumor shows areas of necrosis, haemorrhage and hyalinisation.

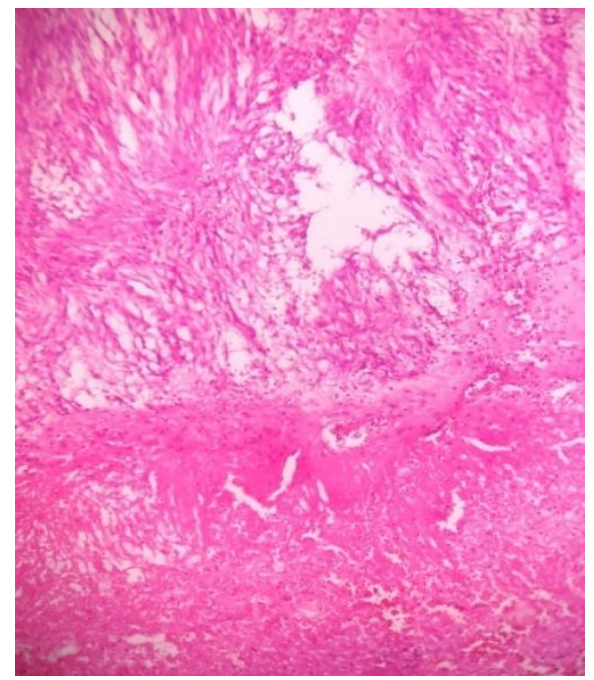

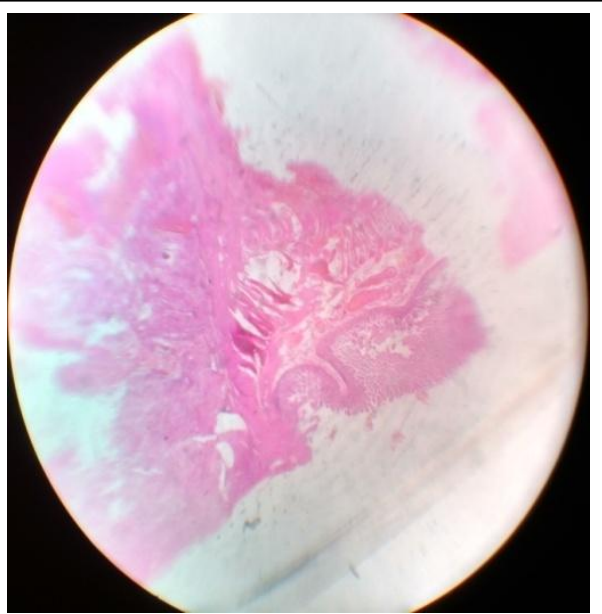

An impression of malignant mesenchymal tumour suggestive of recurrent gastrointestinal stromal tumor (High Grade) was arrived at.

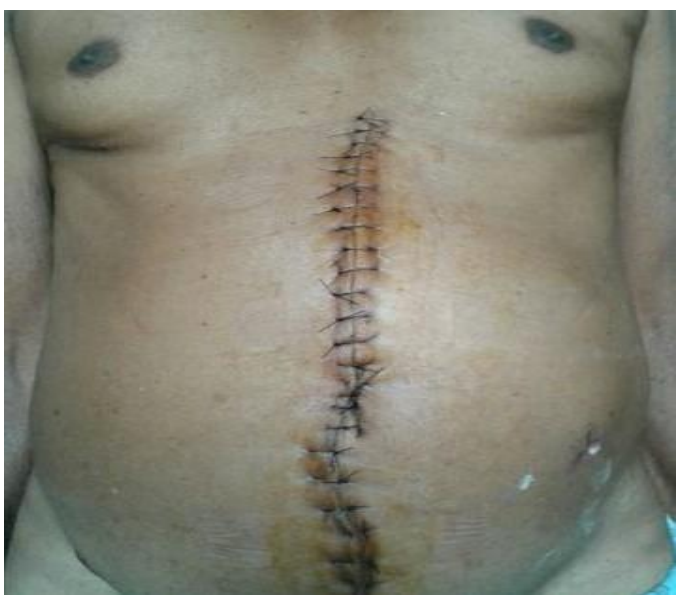

\section{Post operative Image}

Immunohisto chemistry: CD 117 was performed and reported positive

\section{Discussion}

Gastrointestinal stromal tumors (GISTs) are the most common mesenchymal neoplasms of the gastrointestinal tract. They may also originate in the mesentery and omentum. Overall, GISTs are rare and rank a distant third in prevalence behind adenocarcinomas and lymphomas among the histologic types of gastrointestinal tract tumors ${ }^{1}$.

Historically, these lesions were classified as leiomyomas or leiomyosarcomas as they possessed smooth muscle features when examined under microscopy. GISTs are usually found in adults between ages 40 and 70 and can be malignant or noncancerous ${ }^{2}$.

GIST can occur anywhere along the GI tract, most often are found in the stomach or small intestine. 
The American Joint Committee on Cancer (AJCC) Cancer Staging Manual lists the following approximate distributions: ${ }^{3}$

- Stomach $(60 \%)$.

- Small intestine (30\%).

- Rectum (3\%).

- Colon (1-2\%).

- Oesophagus $(<1 \%)$.

- Omentum/mesentery (rare)

Infrequently, a gastrointestinal stromal tumour may arise in the appendix, gallbladder, pancreas, retroperitoneum, and paravaginal and periprostatic tissues. ${ }^{4}$ Nearly $20 \%$ to $25 \%$ of gastric GIST and $40 \%$ to $50 \%$ of small intestinal GIST are clinically aggressive. $^{5,6}$ It has been estimated that approximately $10 \%$ to $25 \%$ of patients present with metastatic disease. ${ }^{5,7}$

These neoplasms range in size from less than $1 \mathrm{~cm}$ to more than $40 \mathrm{~cm}^{8}$ Small GISTs may form solid subserosal, intramural, or, less frequently, polypoid intraluminal masses. Large tumours tend to form external masses attached to the outer aspect of the gut involving the muscular layers. ${ }^{2}$

GIST morphology is quite varied; the tumours are composed of the following: ${ }^{9}$

- Spindle cells (70\%).

- Epithelioid cells (20\%).

- Mixed spindle and epithelioid cells (10\%). Approximately $85 \%$ of GIST contain oncogenic mutations in one of two receptor tyrosine kinases: KIT or PDGFRA (platelet-derived growth factor receptor alpha). ${ }^{8}$ Constitutive activation of either of these receptor tyrosine kinases plays a central role in the pathogenesis of GIST. ${ }^{8,10}$ Wild-type tumours, with no detectable KIT or PDGFRA mutations, account for $12 \%$ to $15 \%$ of all GIST. Fewer than $5 \%$ of GIST occur in the setting of syndromic diseases, such as neurofibromatosis type 1 (NF1), Carney triad syndrome, and other familial diseases. ${ }^{8,11-13}$

A mutation in the kit gene located on chromosome 4 has been implicated in the causation of these tumours. This gene encodes a transmembrane protein which serves as a receptor for the growth factor called stem cell factor (SCF). The transmembrane protein has an intracellular domain containing the tyrosine kinase enzyme, which, once activated, propagates a series of activities ultimately causing cell division.

The mutation in the kit gene causes uncontrolled activation of the tyrosine kinase independently of the growth factor SCF. Activation of tyrosine kinase involves the binding of an adenosine triphosphate molecule to the active site of the tyrosine kinase. ${ }^{14}$

Evaluation of a patient presenting with a GIST should be aimed at pathological confirmation of the diagnosis along with accurate staging of the disease. Tests which need to be done in all patients essentially include:

- Standard white light endoscopy with 6-8 biopsies from the tumor for confirmation of the diagnosis (c-kit/ DOG-1 testing on immunohistochemistry). ${ }^{15}$

- CT scan (multi-detector or helical) of the abdomen and pelvis which consists of a non enhanced phase, an arterial phase, and a portal venous phase. Patients may receive a negative/water-equivalent oral contrast agent for the detection of GI tract wall lesions. If the patient is allergic to contrast media, then an MRI of the abdomen is recommended along with a non contrast CT thorax.

- CT Thorax or X-ray for staging of the chest, and Routine blood investigationscomplete blood counts, renal function tests, and liver function tests.

Optional investigations when indicated include:

- 2-[18F] fluoro 2-deoxyD-glucose-PET or PET-CT-if metastatic disease is suspected.

- EUS may be used for gastric GISTs to stage and accurately identify subjects with an early GIST in whom endoscopic therapy could be planned.

The most frequently used tumour marker for GIST is the CD117 antigen, a marker expressed by ICC. Approximately $95 \%$ of GISTs are positive for the CD117 antigen, an epitope of the KIT receptor tyrosine kinase. ${ }^{8}$ 
In patients presenting with a primary GIST, only complete surgical resection offers a possibility of cure. Although surgery has a high rate of cure for low-risk GIST, it rarely has a curative intent in high-risk tumors since the postoperative recurrence rate for GIST with high-risk features is very high.

Imatinib Mesylate is now a cornerstone in the treatment of GISTs. It is a 2phenylaminopyrimidine derivative. Administered only orally, it has an oral availability of $98 \%$ in patients with normal hepatic function. Its absorption is not modified by food. It binds primarily to the albumin in plasma. Peak plasma levels occur 2-4 hours after oral administration. ${ }^{17}$ It is metabolized mainly by the cytochrome P450 system in the gut wall and liver. Its major metabolite is the $\mathrm{N}$-demethylatedpiperazine derivate, which is also active. The elimination half-life for IM and its active metabolite averages 18 and 40 hours, respectively.

IM is mainly excreted by fecal elimination via the bile, mostly as metabolites. A quarter of the dose is excreted unchanged. Age does not seem to have any significant effect on pharmacokinetics. The dosage needs to be adjusted in severe hepatic or renal impairment. ${ }^{14,16}$ the most common toxic effects of IM at $400 \mathrm{mg}$ once daily included nausea, vomiting, edema, skin rash, and bone marrow toxicity. These side effects were reported to be tolerable. A small percentage of patients with large bulky tumors experienced significant tumor hemorrhage. ${ }^{17}$

The risk of postoperative recurrence was fairly high in tumors with high-risk features in a study conducted by Pierie et al which demonstrated a 5year overall survival of $42 \%$ in GIST patients with complete gross resection compared with $9 \%$ if the excision was incomplete.

Another study by DeMatteo confirmed a similar5year disease-specific survival of $35 \%$ in 200 patients who had surgical resection for primary or metastatic GIST. ${ }^{18}$

In patients with locally advanced disease, surgical resection can be undertaken following neoadjuvant Imatinib. Preoperative imatinib may be used in the following settings: If an R0 resection is unlikely, very large localized, but potentially resectable GIST that may bleed or rupture, poorly located small GISTs that are difficult to resect, non metastatic, but localized, GISTs deemed unresectable. The optimal duration of preoperative therapy is unknown; hence, imatinib may be continued until maximal response. Early response assessment after 8 weeks of initiation of therapy should be done.

\section{Conclusion}

Management of a patient diagnosed with GIST presents to the surgeon with management options including but not limited to surgery and Imatinib Mesylate therapy which are two powerful methods that help prognosticate long term survival. For primary resectable GIST, the first step should include always complete excision of the tumor.

Neoadjuvant IM therapy can be considered in a subset of patients with an initially unresectable tumor or if a downsizing of the tumor would allow resection with decreased intra and post operative surgical morbidity. Adjuvant Imatinib Mesylate should be given to patients at higher risk of recurrence.

In patients with metastatic or recurrent GIST, the treatment is based primarily on Imatinib Mesylate therapy and for resistant or intolerant patients, Sumatinib Mesylate can be used A multidisciplinary approach and close collaboration between a medical oncologist, gastroenterologist, radiologist, and principally the surgeon are mandatory to provide GIST patients with the best available treatment.

This case is particularly rare and notable as the patient remained asymptomatic after first surgery for a period of 9 years with high grade gastro intestinal stromal tumor and arising from mesentery which is an unusual presentation. 


\section{References}

1. Kindblom LG, Remotti HE, Aldenborg F, Meis-Kindblom JM. Gastrointestinal pacemaker cell tumor (GIPACT): gastrointestinal stromal tumors show phenotypic characteristics of the interstitial cells of Cajal. Am J Pathol. 1998;152:1259- 1269.

2. Hirota $\mathrm{S}$, Isozaki $\mathrm{K}$, Moriyama $\mathrm{Y}$, et al. Gain-of-function mutations of c-kit in human gastrointestinal stromal tumors. Science. 1998;279:577-580.

3. Gastrointestinal stromal tumor. In: Edge SB, Byrd DR, Compton CC, et al., eds.: AJCC Cancer Staging Manual. 7th ed. NewYork, NY: Springer, 2010, pp 175-80.

4. Corless CL, Fletcher JA, Heinrich MC: Biology of gastrointestinal stromal tumors. J ClinOncol 22 (18): 3813-25, 2004.

5. Joensuu H: Gastrointestinal stromal tumor (GIST). Ann Oncol 17 (Suppl 10): x280-6, 2006.

6. Miettinen M, Lasota J: Gastrointestinal stromal tumors: review on morphology, molecular pathology, prognosis, and differential diagnosis. Arch Pathol Lab Med 130 (10): 1466-78, 2006.

7. DeMatteo RP, Lewis JJ, Leung D, et al.: Two hundred gastrointestinal stromal tumors: recurrence patterns and prognostic factors for survival. Ann Surg 231 (1): 518, 2000.

8. Corless CL, McGreevey L, Haley A, Town A, Heinrich MC. KIT mutations are common in incidental gastrointestinal stromal tumors one centimeter or less in size. Am J Pathol. 2002;160:1567-1572.

9. Heinrich $\mathrm{MC}$, Blanke $\mathrm{CD}$, Druker $\mathrm{BJ}$, Corless CL. Inhibition of KIT tyrosine kinase activity: a novel molecular approach to the treatment of KIT-positive malignancies. J ClinOncol. 2002;20:16921703.
10. Hirota S, Isozaki K, Moriyama Y, et al.: Gain-of-function mutations of c-kit in human gastrointestinal stromal tumors. Science 279 (5350): 577-80, 1998.

11. Andersson J, Sihto H, Meis-Kindblom JM, et al.: NF1-associated gastrointestinal stromal tumors have unique clinical, phenotypic, and genotypic characteristics. Am J SurgPathol 29 (9): 1170-6, 2005.

12. Agaimy A, Pelz AF, Corless CL, et al.: Epithelioid gastric stromal tumours of the antrum in young females with the Carney triad: a report of three new cases with mutational analysis and comparative genomic hybridization. Oncol Rep 18 (1): 9-15, 2007.

13. Carney JA: Gastric stromal sarcoma, pulmonary chondroma, and extra-adrenal paraganglioma (Carney Triad): natural history, adrenocortical component, and possible familial occurrence. Mayo ClinProc 74 (6): 543-52, 1999

14. Demetri G, Antonia S, Benjamin R, et al. NCCN Clinical Practice Guidelines in Oncology, Soft Tissue Sarcoma; 2009.

15. Tuveson DA, Willis NA, Jacks $\mathrm{T}$, et al. STI571 inactivation of the gastrointestinal stromal tumor c-KIT oncoprotein: biological and clinical implications. Oncogene. 2001;20:5054- 5058.

16. de Kogel C, Schellens JHM. Imatinib. Oncologist. 2007;12(12): 1390-1394.

17. Demetri G, von Mehren M, Blanke C, et al. Efficacy and safety of imatinibmesylate in advanced gastrointestinal stromal tumors. N EnglJ Med. 2002;347(7):472480.

18. DeMatteo R, Lewis J, Leung D, Mudan S, Woodruff J, Brennan M. Two hundred gastrointestinal stromal tumors: recurrence patterns and prognostic factors for survival. Ann Surg. 2000;231(1):51-58. 